\title{
N-best evaluation for academic hiring and promotion
}

\author{
Michael C. Frank \\ Department of Psychology, Stanford University
}

Author Note: Please address correspondence to Michael C. Frank, 450 Serra Mall, Stanford, CA 94305. mcfrank@stanford.edu 


\begin{abstract}
How should we evaluate candidates for academic positions? Current evaluations privilege quantity of publications and journal prestige, creating perverse incentives. To avoid this issue, I propose a N-best policy: Hiring and promotion committees should solicit a small number $(\mathrm{N})$ of research products and read them carefully as the primary locus of evaluation, using other elements like letters or job talks to contextualize this work. This policy aligns evaluation more directly with the goal of selecting scientists who produce high quality work.
\end{abstract}




\section{N-best evaluation for academic hiring and promotion}

Before we vote on hiring or promotion decisions, my department conducts a peculiar ritual. A member of the committee must present a set of the candidate's papers - which have been provided to the faculty beforehand - in detail, walking the assembled group through the results. If members of the department have concerns about the candidate's work, these papers are the locus of criticism. Often specific results, particular figures even, are discussed at length. When I first joined the department, I was surprised by this time-consuming and occasionally painful ritual. When we had just seen a job talk and had one-on-one meetings, why shouldn't we begin by sharing our evaluation of the candidate without listening to a summary of specific papers?

Over the last nine years, my perspective has gradually been changing. I've become increasingly aware of what is termed the "credibility crisis," a set of concerns about the published literature in psychology and beyond. In particular, I no longer take for granted that empirical findings can be replicated by independent investigators (Open Science Collaboration, 2015). I also now know that many published statistical analyses cannot be reproduced, even with access to empirical data (Hardwicke et al., 2018). Further, I have heard the ways that researchers talk about their peers using terms like "productive" or "impactful" - terms that function as code words for publishing lots of papers in high-prestige journals. In this context, it is hard not to see the link between the pressure to publish and issues of reproducibility and replicability (Munafó et al., 2017; Nosek et al., 2012). This link even emerges in simulations of the scientific ecosystem, with publication pressure causing an inevitable "natural selection of bad science" (Smaldino \& McElreath, 2016). 
This growing awareness has led me to re-construe my department's ritual. I now see it as an appropriate scoping of discussion - away from relatively irrelevant metrics like the impact factor of the journal in which a paper appeared (Alberts, 2013) and towards the actual content of specific articles. This procedure aligns our process with our goal (Dougherty, Slevic, \& Grand, 2019). Since the goal of hiring and promotion is to find and retain the researchers that produce the best work, we want an evaluation process that identifies such scientists. If our proxy for scientific excellence is publication quantity and journal prestige, we will end up with universities full of people who publish a lot of papers quickly, submitting each one over and over to different selective journals in hopes of getting lucky. In contrast, evaluating individual papers may not be a perfect proxy for scientific excellence, but it will at least select for scientists who have produced solid scholarship.

This specific set of experiences can be formalized as what I'll call the N-Best proposal: Hiring and promotion committees should solicit a small number (N) of research products and read them carefully as the primary metric for evaluation of candidates.

I don't take any credit for this proposal - it has been discussed widely and has clearly informed the design of institutional instruments like the US NIH's biosketch format or the UK's Research Evaluation Framework (REF). The $\mathrm{N}$ in this particular version refers to "products" rather than "papers," because they can be unpublished papers (e.g., preprints) as well as thesis chapters, datasets, or other artifacts like software, when these are appropriate contributions for the position. All other aspects of an applicant's file, including institutional background, lab, other publication record, and especially letters of recommendation or evaluation (which are often 
written by experts in a particular subfield), should serve as context for those pieces of scholarship.

What should $\mathrm{N}$ - the number of products - be? I expect that this number would change substantially depending on field, position, and institution. In the context I know best - cognitive science research at an R1 university - I'll make some suggestions. For postdoctoral fellowships, only one or two products. Having finished two good projects during a $\mathrm{PhD}$ is enough to show that you are able to initiate and complete projects; some PhDs only yield a single product, but typically this product will be quite substantial. For tenure-track positions: three products, enough to give some intimation of a coherent set of methods and interests. A research statement will typically be necessary for contextualizing this work and describing future plans. For promotion reviews, five products. If a candidate has written five really good papers - papers that substantially advance theory or practice - I think they deserve tenure at a major research university. Some of these products could be reviews or theoretical syntheses, giving the opportunity to bring together many empirical contributions.

Under current evaluation standards, the candidate's CV is currently the primary locus of assessment. It's common to ask “does X's CV look good?” Under an N-best policy, the CV - specifically the publications list, which emphasizes quantity and journal placement - is de-emphasized. Instead, the question is "what has X discovered?" or "how has X contributed to the field?" Under current standards, letters of recommendation are typically solicited; guidance is uneven across institutions about whether they should contain holistic evaluations of the candidate or focused interpretation of their scholarship. Under N-Best, the primary and explicitly-stated goal of letters would be to contextualize the candidate's scholarship and its contributions to the 
broader research enterprise, helping to mitigate the problem of assessing work outside of the department's expertise. Other general statements, e.g. about productivity, brilliance, etc. would be discounted (following evidence that they may be vehicles for bias; Leslie et al., 2015). Finally, under current evaluation standards for hiring especially, we weigh job talks and unstructured interviews very heavily, despite the relatively low validity of such practices (e.g., McDaniel et al., 1994; Schmidt \& Hunter, 1998). Under N-best, the goal would be to use interviews/job talks to assess the quality of the submitted research products. If the evaluation of a small number of distinct research findings is the nexus of the assessment, then what someone wore or whether they were "charismatic" (e.g., attractive and friendly) becomes a bit harder to confuse with the task at hand.

A number of objections come to mind. First of all, it is expensive to implement N-best across a large set of candidates; it may be necessary to evaluate the broader pool more superficially, using letters and perhaps a single paper as a guide. Furthermore, high-quality research is in the eye of the beholder. Members of a department will likely be knowledgeable about an idiosyncratic subset of the research literature; outside of this subset they may be biased towards the most accessible work. These biases hold true for journal editors as well, however. Even in the absence of particular domain expertise, evaluators can judge research on the clarity of its logic and design, the precision of its methods, the presence of indicators of research integrity such as open data and reproducible results, and the extent to which it appears to contribute to a broader enterprise. When in doubt, they can rely more heavily on letters or consult informally with external experts. Finally, for some positions, evaluating future promise is perhaps more important than past performance. While past job performance is in general a very 
good predictor of future success (Schmidt \& Hunter, 1998), a research statement contextualizing current work and describing future plans can be a valuable source of insight into the specificity of future research, complementing specific research products.

One further benefit of N-best is that, if we're actually reading a candidate's research, it need not be published in any particular journal: it can easily be a preprint. Under the current publication system, a typical US PhD student will have to have finished a project by the end of their third year or before in order for the paper to be "in press" by the time they are looking for jobs and postdocs. The same is true for tenure candidates, who have to focus on writing several years before being considered so their work is reflected in the printed record. Because of this timing issue, small sample fluctuations caused by slow journals or chance rejections dramatically shape early careers. Allowing preprints lets us evaluate candidates on the work they've done, not the work they've pushed through the highly variable publication process.

But the primary benefit of N-best evaluation is that our metric is tied directly to the quantity that we want to optimize: good science. Our current evaluations privilege people who do things that are only noisily correlated with doing good science - for example, publishing a large number of papers, or publishing in certain journals. Further, our evaluations create an environment where these metrics - rather than the production of real scientific advances - are paramount. In contrast, if we want our institutions to produce quality research, we need to hire and promote people who do it, and we need to create a set of standards that incentivize it. That means that we need to read candidates' papers and use our best judgment in assessing them. 


\section{References}

Alberts, B. (2013). Impact Factor Distortions. Science, 340, 787. doi: 10.1126/science.1240319

Dougherty, M. R., Slevc, L. R., \& Grand, J. A. (2019). Making research evaluation more transparent: Aligning research philosophy, institutional values, and reporting. Perspectives on Psychological Science, 14(3), 361-375.

Hardwicke, T. E., Mathur, M. B., MacDonald, K., Nilsonne, G., Banks, G. C., Kidwell, M. C., ... \& Lenne, R. L. (2018). Data availability, reusability, and analytic reproducibility: Evaluating the impact of a mandatory open data policy at the journal Cognition. Royal Society Open Science, 5(8), 180448.

Leslie, S. J., Cimpian, A., Meyer, M., \& Freeland, E. (2015). Expectations of brilliance underlie gender distributions across academic disciplines. Science, 347(6219), 262-265.

McDaniel, M. A., Whetzel, D. L., Schmidt, F. L., \& Maurer, S. D. (1994). The validity of employment interviews: A comprehensive review and meta-analysis. Journal of Applied Psychology, 79(4), 599.

Munafò, M. R., Nosek, B. A., Bishop, D. V., Button, K. S., Chambers, C. D., Du Sert, N. P., ... \& Ioannidis, J. P. (2017). A manifesto for reproducible science. Nature Human Behaviour, 1(1), 0021.

Nosek, B. A., Spies, J. R., \& Motyl, M. (2012). Scientific utopia: II. Restructuring incentives and practices to promote truth over publishability. Perspectives on Psychological Science, 7(6), 615-631.

Open Science Collaboration. (2015). Estimating the reproducibility of psychological science. Science, 349(6251), aac4716. 
Schmidt, F. L., \& Hunter, J. E. (1998). The validity and utility of selection methods in personnel psychology: Practical and theoretical implications of 85 years of research findings.

Psychological Bulletin, 124(2), 262.

Smaldino, P. E., \& McElreath, R. (2016). The natural selection of bad science. Royal Society Open Science, 3(9), 160384. 